\title{
Design and Experimental Validation of an LTE-based Synchrophasor Network in a Medium Voltage Distribution Grid
}

\author{
Asja Derviškadić*, Paolo Romano*, Chang $\mathrm{Ge}^{\dagger}$, Wei Koong Chai ${ }^{\ddagger}$, \\ Chris Develder ${ }^{\S}$, Lorenzo Zanni*, Marco Pignati* and Mario Paolone* \\ * Distributed Electrical Systems Laboratory, EPFL, Lausanne, Switzerland \\ $\dagger$ 5GIC, Institute for Communication Systems, University of Surrey, Guildford, United Kingdom \\ $\ddagger$ Department of Computing and Informatics, Bournemouth University, Dorset, United Kingdom \\ $\S$ IDLab, Dept. of Information Technology, Ghent University - imec, Ghent, Belgium
}

\begin{abstract}
We present and experimentally validate in a realscale medium voltage (MV) grid a synchrophasor network that exploits the availability of a public 4G LTE communication infrastructure. An 18 buses, $10 \mathrm{kV}$ feeder located in Huissen, The Netherlands, has been equipped with 10 Phasor Measurement Units (PMUs) connected to the MV grid by means of dedicated voltage and current sensors. The PMUs stream synchrophasor data through a public 4G LTE network via an informationcentric networking-based middleware, named C-DAX. The measurements are received and time-aligned at a phasor data concentrator and fed to a real-time state estimation application. The paper presents the various field-trial components and validates the feasibility of exploiting the $4 \mathrm{G}$ LTE technology for PMUbased applications. Specifically we assess the performance of the adopted wireless telecommunication infrastructure with and without the C-DAX middleware, as well as the accuracy of the real-time state estimation process.
\end{abstract}

Index Terms-4G LTE, Phasor Measurement Unit (PMU), Synchrophasors.

\section{INTRODUCTION}

Monitoring and automation at the power distribution level are growing in importance as distribution grids are evolving from purely passive to active systems. Among the various technologies, Phasor Measurement Units (PMUs) have raised the interest of several distribution network operators (DNOs) to develop advanced control and protection mechanisms [1].

Synchrophasor data must be streamed from PMUs to Phasor Data Concentrators (PDCs) through a telecommunication infrastructure characterized by sufficiently high bandwidth, low end-to-end latency and limited packet loss. The IEEE Std. C37.118.2 [2] does not impose any restriction on the adopted communication system or media. For security and reliability reasons, the favorite solution for many electrical utilities is to use their private legacy wired networks (e.g., fiber links), that however cover high-voltage (HV) transmission

This work was supported by the European Communitys Seventh Framework Programme under Grant FP7-ICT-2011-8 and Grant Agreement 318708 (CDAX) grids only. Moreover, large-scale wired-line based communication infrastructures are not flexible enough to easily support grid topology upgrades. Consequently, DNOs look for costeffective and flexible solutions to transmit data in real-time also in medium-voltage (MV) and low-voltage (LV) grids.

Recently, the use of third-party $4 \mathrm{G}$ Long-Term Evolution (LTE) wireless communication networks has raised the interest of DNOs. Compared to the deployment of an equivalent wired infrastructure, LTE's main advantage is the massive reduction of the related installation costs and time. However, this comes at the price of relying on a publicly shared infrastructure, designed for best effort service support of voice and data applications, without any service level agreement (SLA) with the mobile network provider. As such, it is characterized by relatively high delays, non-deterministic latency variations, packet reordering and risks of significant data loss [3]. Clearly, this also implies availability issues (potentially because of, e.g., denial-of-service attacks [4]). We however leave such availability analysis out-of-scope for this paper.

Rather, in this work we study the practical feasibility of using a public commercial 4G LTE wireless network to support synchrophasor data streaming in a real-scale MV grid in The Netherlands. Specifically, 10 PMUs have been installed in different substations within a $10 \mathrm{kV}$ distribution feeder. The PMUs communicate with dedicated industrial-grade $4 \mathrm{G}$ routers that stream synchrophasor data through a local public LTE network to a remote PDC [5]. Also, we experiment and analyze the effects of an information-centric networking (ICN) based communication middleware, called C-DAX [6], [7], [8], in terms of data transfer latency and data loss. CDAX is a topic-based publish-subscriber engine that decouples data producers and consumers in time and space. Finally, synchrophasor measurements are pushed to Real-Time State Estimation (RTSE) that provides a reliable estimate of the grid state. The RTSE is fundamental to compensate for data losses.

We summarize our contributions as follows. We present the deployment of a synchrophasor network over MV distribution grids using a public 4G LTE infrastructure, describing both the general architecture (Section II) and the specific field-trial 


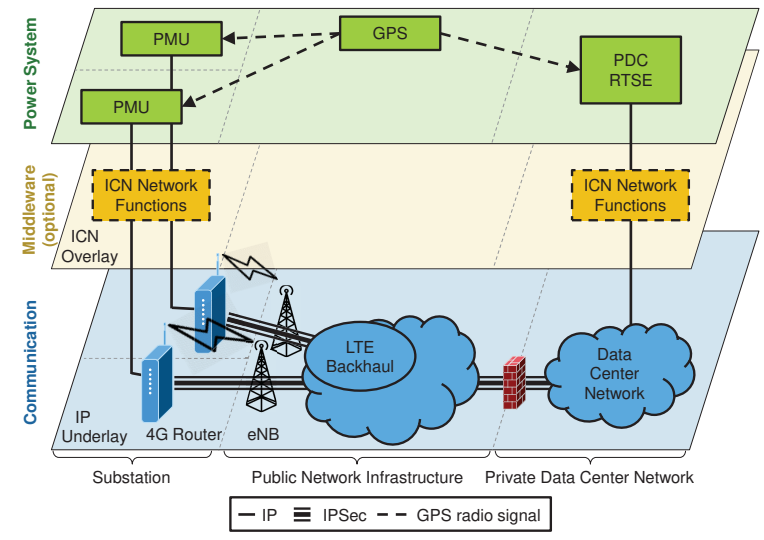

Figure 1. Architecture of a generic PMU deployment in MV grids over a public 4G LTE network, including an optional ICN overlay.

setup (Section III). We assess the performance of the public 4G LTE network in terms of end-to-end latency and data loss (Section IV-A). We compare the performance with and without the C-DAX middleware (Section IV-A). We validate the performance of the RTSE algorithm (Section IV-B).

\section{System Architecture}

A generic PMU-deployment in MV grids, leveraging on a 4G LTE network to stream synchrophasor data, can be vertically split into three layers: power system, communication and an optional middleware layer (see Fig. 1).

\section{A. Power System Layer}

The power system layer comprises the PMUs, that are installed in MV substations, and the PDC, that is typically installed in dedicated servers at the DNO's data center. Both PMUs and PDC are synchronized to a common time reference that is typically provided by the Global Positioning System (GPS) [9]. The PDC can supply various PMU-based applications, that are typically based on common knowledge of the power system state, which can be determined by wellestablished state estimation (SE) processes. State estimation involves solving an optimization problem that processes the raw measurements and the network model (i.e., the combination of network topology and electrical parameters of the network components) with the aim of determining the most likely estimate of the power system state. A measurement infrastructure exclusively composed of PMUs enables linear and non-iterative SE, and allows us to achieve refresh-rates of tens of estimates per second and sub-second time-latency. Thus, PMU technology facilitates RTSE [10].

\section{B. Communication Layer}

The communication layer includes all networking components involved in the synchrophasor data streaming. PMUs transmit synchrophasor data to the PDC through dedicated LTE routers, using the communication protocol defined in the IEEE Std. C37.118.2 [2]. The data is typically received by the PDC through a dedicated Access Point Name (APN) gateway, providing a firewall functionality that ensures secure communications for the DNO's private data center network. In this context, the main challenges faced by DNOs are cyber security and data streaming performance.

Regarding security, PMU data streams conventionally traverse public Internet domains that are owned by third-party Internet service providers (ISPs) rather than infrastructure privately owned by the DNO. To protect synchrophasor data from being eavesdropped, a virtual private network (VPN) tunnel, e.g., Internet Protocol Security (IPsec), is usually established between the substations and the DNO's private data center network. Such VPN tunnel normally begins at the access point of the substation and terminates at a VPN concentrator inside the data center network.

Regarding performance, the nature of the 4G LTE network itself implies reduced reliability and non-deterministic latency. Specifically, due to the mismatch between the buffer management at radio resource control (RRC) layer, packet data convergence protocol (PDCP) layer and transport layer (TCP/UDP), network latency often exhibits a relatively high variance (in a spiking manner). Additionally, the data loss over the radio air interface is approximately $0.13 \%$ with hybrid auto repeat request (HARQ) enabled, which may be too high for certain mission-critical grid applications [11]. One possibility for the DNO to achieve acceptable network performance would be to establish an SLA with the mobile network operator. However, this is often costly, which may offset the benefits in using wireless network to save costs.

\section{Middleware Layer}

The middleware layer links the power system components with the communication ones while providing advanced communication features. The motivation for introducing middleware is to offer a technology-agnostic abstraction, thus shielding developers from specific (communication) technological details. Instead of centralized point-to-point platforms such as SCADA, we advocate using ICN as it focuses directly on information/data (i.e., "what") rather than the host locations (i.e., "where"), and offers additional advantages (e.g., in terms of security), as discussed in more detail in [7], [8], [12].

However, communication features placed between the PMUs and the PDC, influence the communication performance. Specifically, in case of ICN, any additional network hops and application layer processing (in an intermediary ICN network function) increase the end-to-end delay and jitter. Both factors could impact delay-sensitive applications.

\section{THE FIELD TRIAL}

This Section presents the various field-trial components. First the selected MV feeder is described. Then the synchrophasor network composed of PMUs, PDC and the RTSE application is introduced together with some technical details on their implementation and deployment. Finally the public 4G LTE network and the ICN infrastructure are presented in terms of their components and topology. 


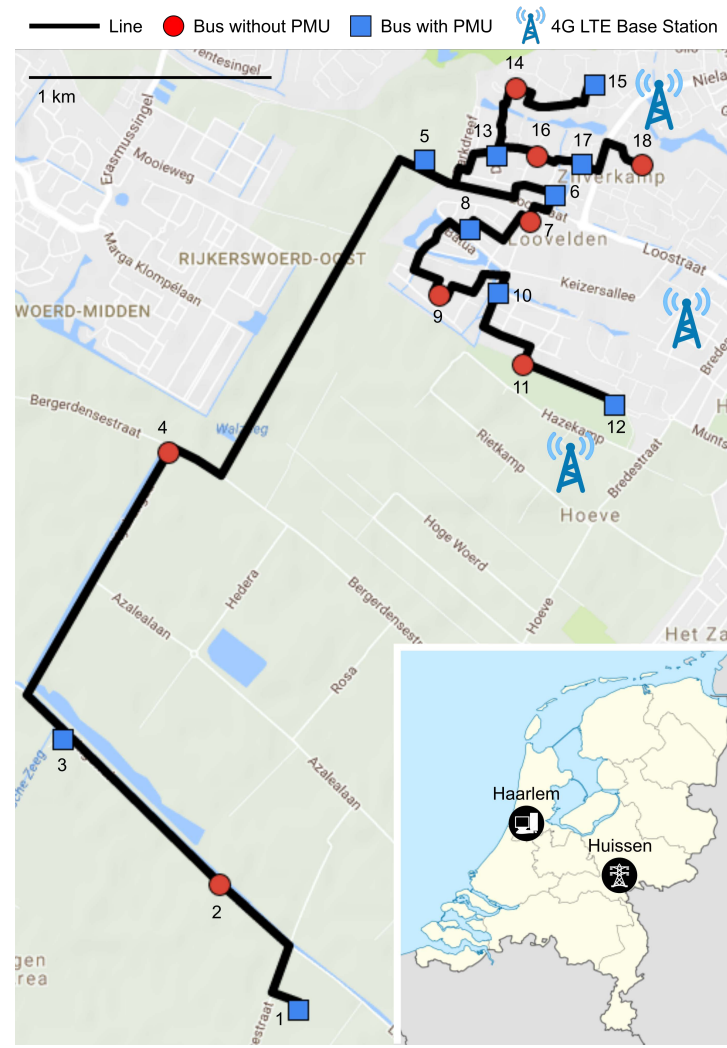

Figure 2. The topology of the field trial (adapted from [5]).

\section{A. The Electrical Grid}

The field trial has been rolled out on a feeder operated by the Dutch DNO Alliander NV, and is considered representative of typical operating conditions of present-day MV distribution networks. The feeder, shown in Fig. 2, is located close to the city of Huissen, The Netherlands, and characterized by a nominal voltage of $10 \mathrm{kV}$. It is composed of a primary substation (node 1) and 17 secondary substations that supply both a rural and an urban area. The former includes 3 medium-size farms, whereas the latter feeds approximately 700 households and a small company. Each secondary substation is equipped with a single $\mathrm{MV} / \mathrm{LV}$ transformer characterized by a power varying from $240 \mathrm{kVA}$ up to $1 \mathrm{MVA}$. The substations are linked through three-phase underground cables with lengths between 218 and $1813 \mathrm{~m}$. The urban area, on the low voltage side, comprises a total of $100 \mathrm{~kW}$-peak of $\mathrm{PV}$, around 35 private and public electric vehicles charging stations and a combined heat power plant with a rated power of $400 \mathrm{kVA}$.

\section{$B$. The Sensing infrastructure}

The sensing infrastructure is composed of 10 PMUs measuring three-phase nodal voltages and injected currents in the nodes represented by a square in Fig. 2. The measurements' locations have been selected to satisfy the full-rank constraint of the state estimator measurement model in order to enforce the grid observability [13].

In the 9 monitored secondary substations, PMUs are interfaced to the MV grid by means of dedicated 0.1 class voltage and current sensors, based on capacitive divider and Rogowski coil principles respectively [14]. In the primary substation (node 1 in Fig. 2), a PMU measures the nodal voltage and the total current absorbed by the feeder by taking advantage of the already installed voltage and current transformers (rated accuracy class 0.5 and 1 respectively).

The PMUs are based on the National Instrument compactRIO platform and integrate the synchrophasor estimation algorithm presented in [15]. Absolute time synchronization is achieved through a stationary GPS receiver characterized by a maximum synchronization uncertainty of $100 \mathrm{~ns}$, connected to a dedicated GPS antenna mounted on the rooftop of each substation. Such a PMU, compliant with all P-class requirements and most of the M-class ones, is characterized by a maximum total vector error (TVE) of $0.02 \%$ that is maintained in both steady-state and most of the dynamic conditions, irrespective of the harmonic-distortion levels. The PMU measurement reporting latency is of $37 \mathrm{~ms}$, independently of the specific operating conditions. It should be noted that, by comparing the rated TVE of the PMU and the nominal accuracy of the sensors, the measurement uncertainty is dominated by the sensors inaccuracies.

\section{Phasor Data Concentration}

The PDC runs on a dedicated server hosted in the Alliander data center, located in the city of Haarlem, around $100 \mathrm{~km}$ away from the PMU installations (see Fig. 2). The server is equipped with an Intel Xeon CPU E5-2630L @ $2.00 \mathrm{GHz}$ (64 bit) and $64 \mathrm{GiB}$ of RAM and runs Red Hat Enterprise Linux (RHEL) distribution. The PDC receives an absolute UTC time reference from a GPS-based master clock that disseminates time through an IEEE 1588 Precision Time Protocol (PTP) with a sub-millisecond uncertainty. The master clock is integrated into a time server installed in the Alliander data center that is connected to a local network through a dedicated PTP switch to which the PDC is connected.

The PDC architecture is presented in [5] and embeds a fixed-size circular buffer implementing two different data pushing logic: an absolute time logic, where the data pushing is performed once a specific UTC time is reached, and a relative time logic, in which the $\mathrm{PDC}$ waits for a specified relative time triggered by an event. The PDC buffer is meant to mitigate the synchrophasor data latency variance introduced by the telecommunication network. In particular, when dealing with public 4G LTE networks, the presence of multiple paths characterized by different latencies throughout the network might cause the so called data reordering effect. This causes data frames generated with consecutive timestamps to reach the PDC out of order. By adopting such a time-alignment buffer, the PDC can automatically recover the original order of timestamps by simply making the buffer big enough to deal with $4 \mathrm{G}$ latency variations.

\section{Real-time State Estimation}

The RTSE is installed in the same server where the PDC runs. Its role is to ensure a complete view of the grid even in 
case of data losses, thanks to measurement redundancy. Every time a new time-aligned dataset is pushed by the PDC, the RTSE calculates the most likelihood network state by means of a Linear Weighted Least Squares (LWLS) method [10]. Let us consider a power system composed of $S$ buses. The system state $\mathbf{x} \in \mathbb{R}^{n}$ is composed of the real and imaginary parts of the bus phase-to-ground voltage phasors in every phase $(a, b, c)$ and at every bus $i \in[1, s]$, being $n=3 \cdot 2 \cdot s$ :

$$
\mathbf{x}=\left[\mathbf{V}_{1, a b c}^{r e}, \ldots, \mathbf{V}_{s, a b c}^{r e}, \mathbf{V}_{1, a b c}^{i m}, \ldots, \mathbf{V}_{s, a b c}^{i m}\right]
$$

As known, the use of synchrophasor measurements makes the measurement model linking the measurement vector $\mathbf{z} \in \mathbb{R}^{m}$ and the system state $\mathbf{x}$ linear:

$$
\mathbf{z}=\mathbf{H x}+\mathbf{w}
$$

being $\mathbf{H} \in \mathbb{R}^{m \times n}$ the measurement matrix and $\mathbf{w} \in \mathbb{R}^{m}$ the measurement noise vector that is assumed to be a white Gaussian sequence with covariance matrix $\mathbf{R} \in \mathbb{R}^{m \times m}$. The measurement uncertainty is typically expressed with magnitude and phase-angle, but the phasor measurements are expressed in rectangular coordinates; therefore, we project the uncertainty from polar to rectangular coordinates by using the procedure reported in [16]. It is worth pointing out that $\mathbf{H}$ which is composed of zeros, ones and elements of the network admittance matrix - is exact and constant. Consequently, for a PMU-only RTSE, the LWLS solution does not require any iteration and is computationally efficient.

The LWLS state estimator consists of an optimization problem that minimizes the following objective function:

$$
J=(\mathbf{z}-\mathbf{H} \mathbf{x})^{T} \mathbf{R}^{-1}(\mathbf{z}-\mathbf{H} \mathbf{x})
$$

The estimated network state $\widehat{\mathbf{x}}$ is computed as:

$$
\widehat{\mathbf{x}}=\left(\mathbf{H}^{T} \mathbf{R}^{-1} \mathbf{H}\right)^{-1} \mathbf{H}^{T} \mathbf{R}^{-1} \mathbf{z}
$$

See [10] for details of the LWLS problem and its solution.

In the field trial the system is composed of $s=18$ buses and the SE is computed using the nodal voltage and nodal injected current measurements generated by the 10 installed PMUs. This leads to a relatively low SE redundancy, that is defined as the ratio between number of measurements and number of state variables, which amounts to $2 \cdot 10 / 18=1.11$. It is important to increase the measurement redundancy level in order to satisfy the observability criteria even in case of missing measurements. To do so, we use also the socalled pseudo-measurements, defined as measurements with large uncertainties that represent predictions typically based on historical data or forecasts. The measurement vector $\mathbf{z}$ becomes [13]:

$$
\mathbf{z}=\left[\mathbf{z}_{V}^{P M U}, \mathbf{z}_{I}^{P M U}, \mathbf{z}_{I}^{\text {pseudo }}\right]
$$

The first two sub-arrays correspond to the synchrophasor measurements of nodal voltages and nodal injected currents in buses $\{1,3,5,6,8,10,12,13,15,17\}$. The third subarray corresponds to the pseudo-measurements of nodal injected currents at the buses not equipped with PMUs, i.e.,
$\{2,4,7,9,11,14,16,18\}$. Since no information is given about the load power absorbed at these buses, at every time-step pseudo-measurements of injected currents are artificially generated in order to cover the maximum power that could be absorbed, which is intuitively equal to the rated power of the MV/LV transformer. Consequently, the pseudo-measurement uncertainties in the covariance matrix $\mathbf{R}$ are calculated in order to cover up to the rated value of the transformer current. These variances are typically two orders of magnitude higher than those associated with real-time current measurements.

\section{E. The Communication Infrastructure}

The telecommunication infrastructure is required to deliver the synchrophasor measurements from PMUs deployed at different substations to the PDC located at Alliander's data center within very stringent latency constraints. In this respect, every PMU reports synchrophasor measurement at a rate of 50 frames per second (fps), a typical value for PMU applications [9], through a dedicated industrial-grade $4 \mathrm{G}$ router Garderos R-2228 [17].

The $4 \mathrm{G}$ routers are connected to a local public $4 \mathrm{G}$ LTE network operated by Vodafone Libertel B.V. in The Netherlands. Each $4 \mathrm{G}$ router connects to the LTE base stations (eNBs) with the highest signal strength (depending on Vodafone's configurations) rather than the closest one (see Fig. 2). As such, the specific eNB that each $4 \mathrm{G}$ router is connected to may change over time. In addition, we use public off-theshelf SIM cards for all $104 \mathrm{G}$ routers. Hence, no specific SLA has been established with the $4 \mathrm{G}$ network operator to guarantee delivery performance. The performance of the $4 \mathrm{G}$ LTE network is thus subject to variations depending on the demand and operating conditions during our experiments. These two factors contribute to uncertainties regarding the latency achieved within our field trial.

An IPSec tunnel is established between each PMU's 4G router and Alliander's data center to ensure secure encrypted data transmission. We use Vodafone's VPN service because it facilitates bandwidth allocation: each $4 \mathrm{G}$ router is allocated with $100 \mathrm{kbps}$ uplink bandwidth at the ingress point of the VPN tunnel, and the PDC server is allocated with $2 \mathrm{Mbps}$ downlink bandwidth at the egress point of the tunnel. However, despite the bandwidth allocation, there is no guarantee on any other network Quality-of-Service (QoS) parameters, especially latency.

As discussed in Section II-C, we also deploy C-DAX as an optional middleware platform in the field trial. Each PMU contains a C-DAX publisher module, which communicates with a single subscriber module at the PDC server via the LTE network.

\section{Performance Assessment}

This Section validates the feasibility of the proposed approach in real operating conditions by assessing the telecommunication infrastructure performance in Section IV-A and the RTSE accuracy in Section IV-B. 


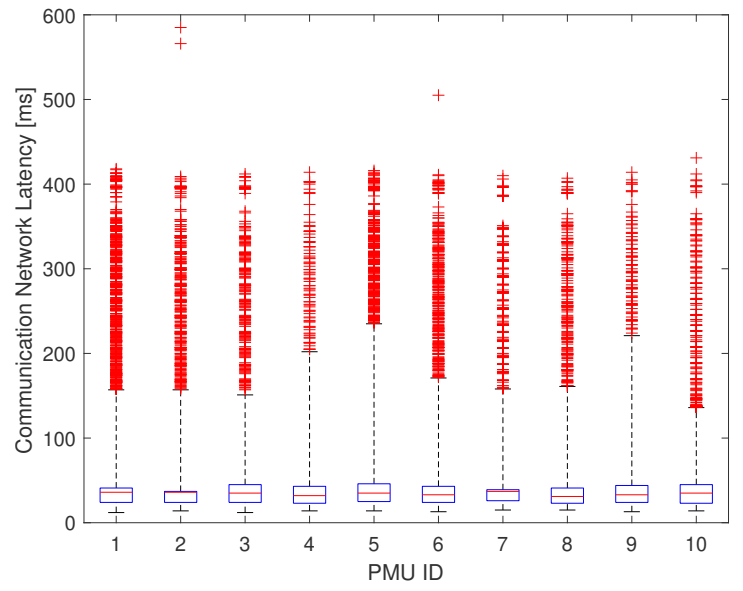

Figure 3. Boxplot representation of the communication network latency per PMU data stream during 24-hours (Jan 31, 2016). The bottom and top edges of the boxes have been modified to indicate the 10th and 90th percentiles respectively, whereas the maximum whisker length is specified as 10 times the interquartile range.

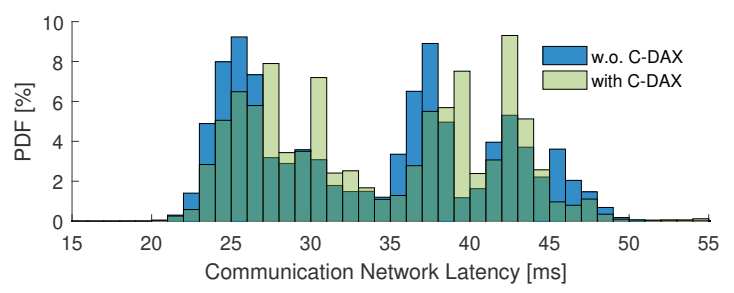

Figure 4. Communication network latency aggregated across all PMU flows during two different days: comparison with (Feb 21, 2016) and without CDAX (Jan 31, 2016)

\section{A. 4G LTE Network Performance}

The performance of the $4 \mathrm{G}$ LTE telecommunication infrastructure is assessed in terms of (1) latency, (2) data loss and (3) dataset completeness. In order to gather the highest amount of PMU data frames regardless of the $4 \mathrm{G}$ network conditions, data has been collected by the PDC using the absolute time logic with a PDC wait time of 1 second. In other words, data frames reaching the PDC with a synchrophasor latency higher than 1 second are discarded and considered as lost packets.

1) Latency: The communication network latency is extremely important when designing a synchrophasor network as it mainly defines the type of power system applications that can run on top of this infrastructure [18]. In this respect, Fig. 3 presents a boxplot of the end-to-end latency measured over 24hours, across all PMU data streams, on January $31^{\text {st }}$, 2016. As can be noticed, the vast majority of values is concentrated in the 20-50 ms range with a median value that is lower than 37 ms and more than $90 \%$ of data received within $45 \mathrm{~ms}$. Figure 3 further shows several outliers in the range of several hundreds of milliseconds.

Figure 4 compares the aggregated end-to-end delay with and without the C-DAX middleware, measured on February $21^{\text {st }}$ and January $31^{\text {st }}, 2016$ respectively. The measurements reveal that the latency distribution is bimodal and show the limited overhead introduced by the C-DAX middleware.

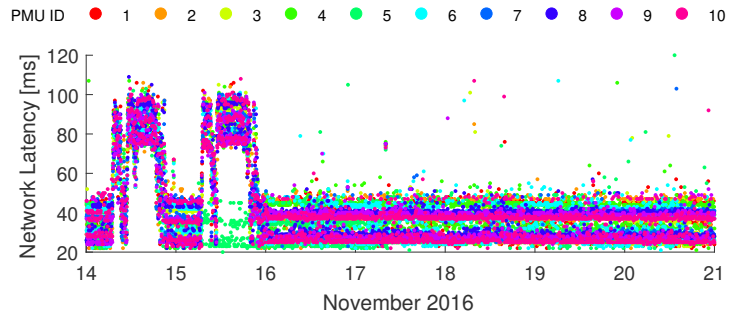

Figure 5. Communication network latency per PMU data stream during 7 days (Nov 14-20, 2016), down-sampled at 1 frame per 5 minutes.

TABLE I

PERCENTAGE OF DAILY DATA LOSS FOR EACH PMU FLOW.

\begin{tabular}{ccccccccccc}
\hline \multicolumn{11}{c}{ Data loss [\%] } \\
\hline \multirow{2}{*}{ day } & 1 & 2 & 3 & 4 & 5 & 6 & 7 & 8 & 9 & 10 \\
\hline
\end{tabular}

$\begin{array}{lllllllllll}\text { Jan } 31 & 0.003 & 0.007 & 0.002 & 0.002 & 0.003 & 0.003 & 0.002 & 0.002 & 0.002 & 0.002\end{array}$ $\begin{array}{lllllllllll}\text { Feb } 21 & 0.005 & 0.003 & 0.006 & 0.004 & 0.004 & 0.004 & 0.005 & 0.005 & 0.004 & 0.012\end{array}$

\begin{tabular}{lllllllllll}
\hline Nov 14 & 0.63 & 0.19 & 0.19 & 0.19 & 0.19 & 0.19 & 0.19 & 0.19 & 0.19 & 0.20
\end{tabular}

\begin{tabular}{lllllllllll} 
Nov 15 & 1.06 & 0.67 & 0.61 & 0.63 & 0.02 & 0.57 & 0.67 & 0.67 & 0.74 & 0.68 \\
\hline
\end{tabular}

Finally, Fig. 5 demonstrates how the end-to-end delays are considerably affected by the varying conditions of the wireless medium. During the first two days of the week between November $14^{\text {th }}$ and November $20^{\text {th }}, 2016$, the whole set of PMUs exhibits a substantial increment $(50 \mathrm{~ms}$ on average) of the end-to-end latency while returning to normal conditions afterwards.

It is worth pointing out that the measured communication network latency is fully compatible with the performance classes TT0 (>1000 ms: Files, events, log contents), TT1 (1000 ms: Events, alarms), TT2 (500 ms: Operator commands) and TT3 (100 ms: Slow automatic interactions) defined in the IEC Std. 61850-5 [18].

2) Data loss: As the field trial relies on a wireless network, successful transmission of data packets is subject to the dynamic conditions of the wireless medium: several effects (interference/noise etc.) may cause the erroneous transmission of bits. While LTE foresees an Automatic Repeat request (ARQ) scheme for the detection of erroneous bits and the retransmission of a packet at the link layer, it does not suffice to cover all possible bit error rates experienced in practice. Note also that the configuration of ARQ is subject to the QoS scheme established, which in the case of our field trial is simply best effort. Moreover, we stress that the streaming nature of the transmitted data along with the low latency requirements have motivated the use of UDP at the transport layer, implying absence of error control above the link layer.

In this respect, Table I compares 24-hours data loss with and without the C-DAX middleware, measured on February $21^{\text {st }}$ and January $31^{\text {st }}$, 2016 respectively, expressed as a percentage of the total number of packets transmitted by each PMU. In general, the loss rate is considerably low, in the order of $0.001 \%$ of the transmitted packets. A slightly higher data loss value can be observed with the C-DAX middleware. This can 
TABLE II

DATASET COMPLETENESS: \% OF DATASETS COMPOSED OF A GIVEN AMOUNT OF DATA FRAMES PUSHED AT DIFFERENT $T_{P D C}($ JAN 31,2016$)$.

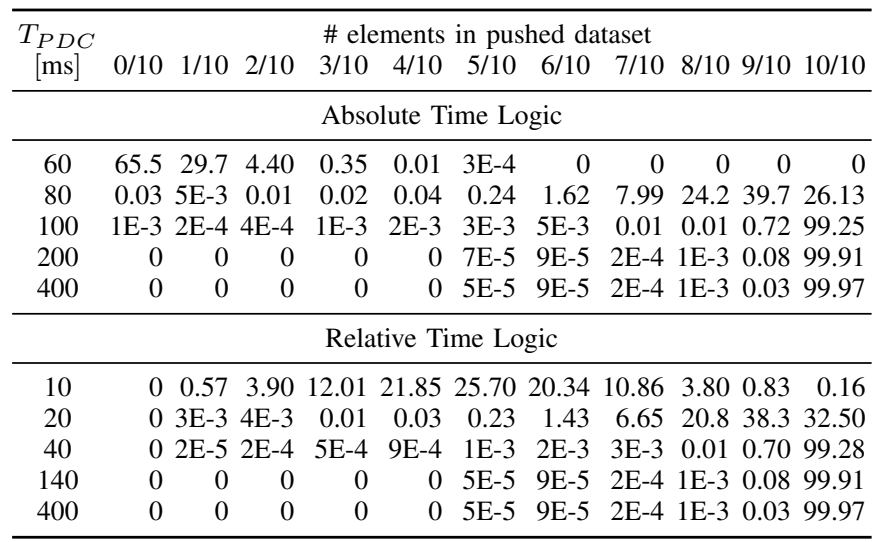

be justified by the C-DAX header overhead which increases the size of the packets and hence also the probability of erroneous packet transmission, given a certain bit error rate. Similarly to the latency analysis, the data loss depends on the specific location of the PMU and on the dynamic conditions of the wireless medium. In this respect, Table I also reports the higher data loss (up to $1 \%$ ) during the network congestion period experienced on November $14^{\text {th }}$ and $15^{\text {th }}, 2016$.

3) Dataset completeness: A PDC collects data frames with the same timestamp into a time-aligned dataset and pushes it to further applications once the specified $P D C$ wait time $T_{P D C}$ elapses, regardless if it is complete or not. In this respect, the dataset completeness, is a key performance index of any synchrophasor network and is influenced by both latency, data loss, and $T_{P D C}$. The latter, in case of absolute time logic, is defined as the difference between the time-aligned dataset push-time and its timestamp. In case of a relative time logic, it is defined as the difference between the time-aligned dataset push-time and the arrival time of its first data frame.

In order to evaluate the effects of $T_{P D C}$ on the dataset completeness, Table II shows completeness statistics for different PDC wait times (absolute and relative time logic). Specifically, when the absolute time logic is adopted, a PDC wait time of $100 \mathrm{~ms}$ enables the forwarding of $99.25 \%$ of complete datasets, whereas in case the relative time logic is considered, a wait time of $40 \mathrm{~ms}$ ensures $99.28 \%$ of complete datasets.

\section{B. Real-Time State Estimation Performance}

In real life, the true state of a real network cannot be known. Indeed, even if we measure the voltage phasors at every bus, measurements are always affected by errors. Therefore, we assess the SE accuracy via offline simulations where the true state of the network is computed and known (e.g., through load flow calculation). The load flow requires the nodal injected power at every bus but the slack bus, and the reference voltage at the slack bus. In order to reproduce realistic conditions, we have used the nodal injected powers and the voltage recorded at $50 \mathrm{fps}$ by the PMUs in the real network. This scenario relies
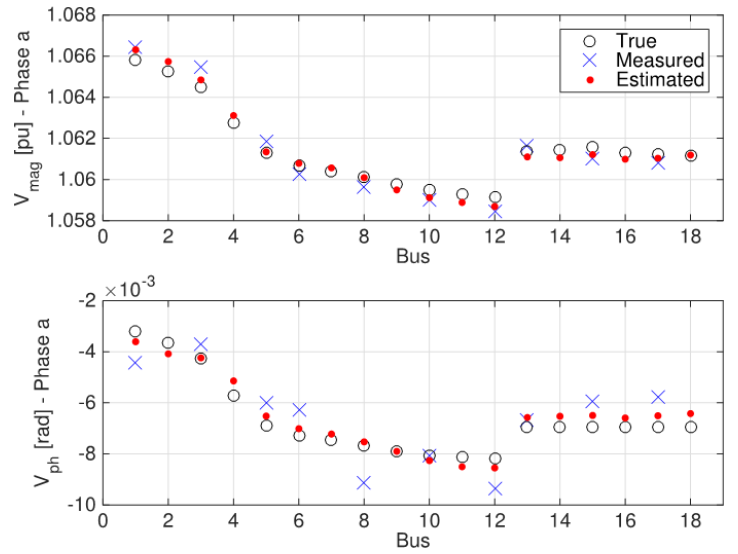

Figure 6. Voltage magnitude and phase at every bus, at time-step 1.
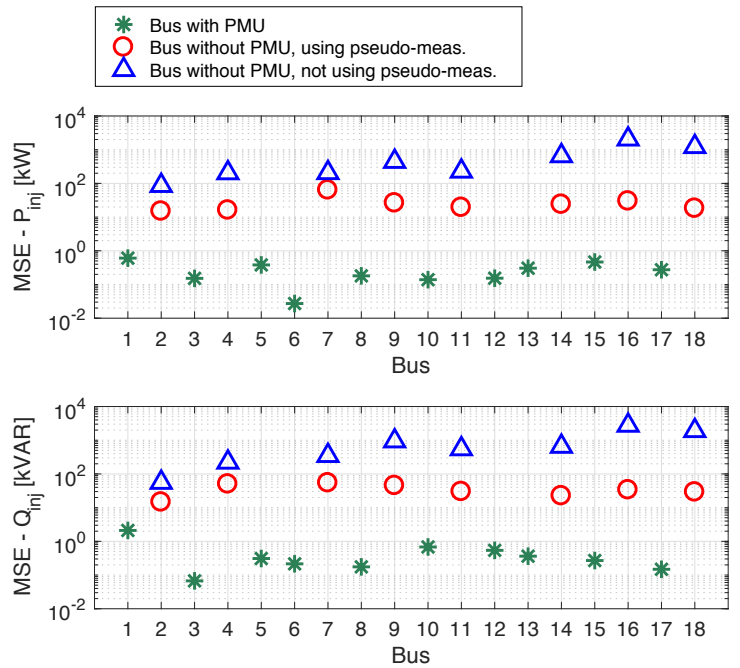

Figure 7. MSEs of the active and reactive injected powers at every bus.

on the assumption that the real measurements are noise-less. The adopted procedure for the offline accuracy assessment of the state estimator is the following:

1) Matrix $\mathbf{H}$ is pre-computed since it remains constant as long as the network topology does not change;

2) For each time step, i.e., every $20 \mathrm{~ms}$, a load flow is computed in order to determine the true state of the network;

3) The synchrophasor measurements at the buses equipped with PMUs are obtained by perturbing the true state of step 1 with: (i) a systematic error as defined by the class of the sensors (see Section III-B) and (ii) a randomly-generated white Gaussian noise characterized by the standard deviation of the noise observed in the real measurements, which is at least a order of magnitude smaller than the sensor error. The pseudo-measurements of injected current are randomly-generated in order to cover the whole power of the MV/LV transformer and by assuming the rated value for the voltage;

4) The elements of matrix $\mathbf{R}$ related to synchrophasor measurements are calculated from the uncertainty defined by 
the sensor's class. The elements of $\mathbf{R}$ related to pseudomeasurements of injected currents are calculated in order to cover the maximum current that can flow through the MV/LV transformer, which is the transformer rated current. Therefore, the pseudo-measurement variances are typically two orders of magnitude higher than those associated with current synchrophasor measurements.

5) The measurements are then forwarded to the state estimator that computes the system state as in (4).

The results consider 10 s (i.e., $N=500$ time-steps at $50 \mathrm{fps}$ ) at $15 \mathrm{~h}$ of January 18,2016 . The analysis is limited to phase $a$, since similar results hold for all phases.

Figure 6 shows the true, measured and estimated values of the voltage magnitude and phase at every bus. We note that the voltage estimates are close to the true values even though the measurements are more dispersed. Indeed, the state estimator performs a best fit of the measurements.

Figure 7 shows the estimation errors of the active and reactive injected powers at every bus expressed as mean square error (MSE). For instance, the MSE of the active injected power at bus $h$ phase $a$ is computed considering $N$ time steps of the estimated $\widehat{P}_{i n j}^{h, a}$ and true $P_{i n j}^{h, a}$ values as:

$$
\operatorname{MSE}\left(P_{i n j}^{h, a}\right)=\sqrt{\frac{1}{N} \sum_{k=1}^{N}\left(\widehat{P}_{i n j, k}^{h, a}-P_{i n j, k}^{h, a}\right)^{2}}
$$

Obviously, the MSEs of the powers at the buses equipped with a PMU are very small, i.e. below $1 \mathrm{~kW} / \mathrm{kVAR}$. At the buses without a PMU, the MSEs are two orders of magnitude larger, because the pseudo-measurements are characterized by large variances. It is important to note that these errors would be even higher if no pseudo-measurements were used. Therefore, pseudo-measurements help to decrease the current/power estimate errors in the buses without PMUs and, as a consequence, pseudo-measurements improve also the estimated voltages.

Further, Fig. 7 quantifies the SE-accuracy decrease when a PMU packet is lost. The network remains observable thanks to the use of pseudo-measurements. However, the MSE of the nodal power estimates at the bus where that specific PMU is located increases to the values of the buses where pseudomeasurements are used (assuming to replace this lost packet with a pseudo-measurement).

\section{CONCLUSION}

This paper has presented the architecture, setup and experimental validation in a real-scale medium-voltage distribution grids of a synchrophasor network that exploits a public $4 \mathrm{G}$ LTE communication infrastructure. Synchrophasor data are generated by PMUs, collected by a PDC and processed by an RTSE process to obtain a snapshot of the monitored grid at a rate of 50 estimations per second.

The field trial has demonstrated that a public 4G LTE network can be used to support the timely transmission of PMU data. In particular, the measured communication network latency is fully compatible with the performance classes TT0, TT1, TT2 and TT3 defined in the IEC Std. 61850-5 [18]. The minimal additional delay from C-DAX middleware components does not alter these conclusions.

Moreover, we have assessed the RTSE performance, demonstrating that the voltage estimates are closer than the measurements to the true voltages. The use of pseudo-measurements decreases the error of current and power estimates at buses without a PMU, even if these errors remain significantly higher than those at buses with a PMU.

\section{REFERENCES}

[1] J. H. Eto, E. Stewart, T. Smith, M. Buckner, H. Kirkham, F. Tuffner, and D. Schoenwald, Scoping study on research and development priorities for distribution-system phasor measurement units, April 2016.

[2] "IEEE standard for synchrophasor data transfer for power systems," IEEE Std C37.118.2-2011 (Revision of IEEE Std C37.118-2005), pp. 1-53, Dec 2011.

[3] C. Kalalas, L. Thrybom, and J. Alonso-Zarate, "Cellular communications for smart grid neighborhood area networks: A survey," IEEE Access, vol. 4, pp. 1469-1493, 2016.

[4] A. N. Bikos and N. Sklavos, "LTE/SAE security issues on 4G wireless networks," IEEE Security Privacy, vol. 11, no. 2, pp. 55-62, March 2013.

[5] A. Derviškadić, P. Romano, M. Pignati, and M. Paolone, "Architecture and experimental validation of a low-latency phasor data concentrator," IEEE Transactions on Smart Grid, vol. PP, no. 99, pp. 1-1, 2016.

[6] "Cyber-secure data and control cloud for power grids," accessed: 2018-01-09. [Online]. Available: http://cdax.ilabt.imec.be/

[7] K. V. Katsaros, W. K. Chai, N. Wang, G. Pavlou, H. Bontius, and M. Paolone, "Information-centric networking for machine-to-machine data delivery: A case study in smart grid applications," IEEE Network, vol. 28, no. 3, pp. 58-64, May 2014.

[8] W. K. Chai and et Al., "An information-centric communication infrastructure for real-time state estimation of active distribution networks," IEEE Transactions on Smart Grid, vol. 6, no. 4, pp. 2134-2146, July 2015.

[9] "IEEE Standard for Synchrophasor Measurements for Power Systems," IEEE Std C37.118.1-2011 (Revision of IEEE Std C37.118-2005), pp. 1-61, Dec 2011.

[10] S. Sarri, L. Zanni, M. Popovic, J. Y. L. Boudec, and M. Paolone, "Performance assessment of linear state estimators using synchrophasor measurements," IEEE Transactions on Instrumentation and Measurement, vol. 65, no. 3, pp. 535-548, March 2016.

[11] B. Han, F. Qian, S. Hao, and L. Ji, "An anatomy of mobile web performance over multipath TCP," in Proceedings of the 11th ACM Conference on Emerging Networking Experiments and Technologies (CoNEXT). New York, NY, USA: ACM, 2015, pp. 5:1-5:7.

[12] W. K. Chai, M. Georgiades, and S. Spirou, Media Networks: Architectures, Applications, and Standards. M. Hassnaa and Z. Sherali, Ed. CRC Press, 2012, ch. 9: Toward Information-Centric Networking: Research, Standardization, Business, and Migration Challenges.

[13] A. Abur and A. G. Expósito, Power System State Estimation: Theory and Implementation. CRC Press, 2004.

[14] "24 kV current and voltage sensor - current and voltage sensor datasheet," accessed: 2018-01-09. [Online]. Available: http://www. alteasolutions.com/current-voltage-sensors/

[15] P. Romano and M. Paolone, "Enhanced interpolated-DFT for synchrophasor estimation in FPGAs: Theory, implementation, and validation of a PMU prototype," IEEE Trans. Instrum. Meas., vol. 63, no. 12, pp. 2824-2836, Dec. 2014.

[16] M. Paolone, J.-Y. L. Boudec, S. Sarri, and L. Zanni, Advances in Power System Modelling, Control and Stability Analysis. F. Milano, Ed. Edison, NJ, USA: IET, 2015, ch. 6: Static and recursive PMU-based state estimation processes for transmission and distribution power grids.

[17] Garderos, "Garderos R-2200 Series Data Sheet," 2013, accessed: 2016-01-09. [Online]. Available: https://www.garderos.com/products

[18] "IEC Communication Networks and Systems in Substations - part 5: Communication requirements for functions and device models." 61850 5 IEC:2003(E), Jul 2003. 